\title{
Symmetries of SU(2) Skyrmion in Hamiltonian and Lagrangian approaches
}

\author{
Soon-Tae Hong, Yong-Wan Kim and Young-Jai Park \\ Department of Physics and Basic Science Research Institute, \\ Sogang University, C.P.O. Box 1142, Seoul 100-611, Korea
}

August 19, 2018

\begin{abstract}
We apply the Batalin-Fradkin-Tyutin (BFT) method to the $\mathrm{SU}(2)$ Skyrmion to study the full symmetry structure of the model at the first class Hamiltonian level. On the other hand, we also analyze the symmetry structure of the action having the WZ term, which corresponds to this Hamiltonian, in the framework of the Lagrangian approach. Furthermore, following the BFV formalism we derive the BRST invariant gauge fixed Lagrangian from the above extended action.
\end{abstract}

PACS: 11.10.Ef, 11.10.-Z, 12.39.Dc

Keywords: Skyrmions, BFT Hamiltonian formalism, Lagrangian approach 


\section{Introduction}

It is well known that baryons can be obtained from topological solutions, known as $\mathrm{SU}(2)$ Skyrmions, since the homotopy group $\Pi_{3}(S U(2))=Z$ admits fermions [1, 2]. Using the collective coordinates of the isospin rotation of the Skyrmion, Adkins et al. [1] have performed semiclassical quantization to obtain the static properties of baryons within about $30 \%$ of the corresponding experimental data.

On the other hand, in order to quantize physical systems subjective to constraints, Dirac quantization scheme [3] has been widely used. However, whenever we adopt the Dirac method, we frequently meet the problem of operator ordering ambiguity. In order to avoid this problem, Batalin, Fradkin, and Tyutin (BFT) developed a method [4], which converts second class constraints into first class ones by introducing auxiliary fields. Recently, we have clarified the relation between the Dirac scheme and BFT one, which has been obscure and unsettled up to now, in the framework of the $\mathrm{SU}(2)$ Skyrmion model [5].

The motivation of this paper is to systematically apply the BFT and Batalin, Fradkin, and Vilkovisky (BFV)-BRST method to the SU(2) Skyrmion as a phenomenological example of topological system, and to consider the problem of finding all local symmetries of the system through both the Hamiltonian and Lagrangian approaches. As a result, we will show that these approaches give the same symmetry structure of the SU(2) Skyrmion. In section 2, we will briefly recapitulate the construction of the first class $\mathrm{SU}(2)$ Skyrmion Hamiltonian. In section 3, we will study full symmetry structure of the system in the pure Hamiltonian approach recently proposed [6]. In section 4 , we will treat symmetry structure of the Lagrangian, which includes the Wess-Zumino (WZ) term, corresponding to the first class Hamiltonian in the Lagrangian approach, to compare with the results in the previous section. Finally, we will construct the BRST invariant gauge fixed Lagrangian from the extended one corresponding to the first class Hamiltonian in the BFV scheme [7, 8, 9, 10]. 


\section{BFT Hamiltonian for SU(2) Skyrmion}

Now we start with the Skyrmion Lagrangian of the form

$$
L_{S M}=\int \mathrm{d} r^{3}\left[-\frac{f_{\pi}^{2}}{4} \operatorname{tr}\left(\partial_{\mu} U^{\dagger} \partial^{\mu} U\right)+\frac{1}{32 e^{2}} \operatorname{tr}\left[U^{\dagger} \partial_{\mu} U, U^{\dagger} \partial_{\nu} U\right]^{2}\right]
$$

where $f_{\pi}$ is the pion decay constant and $e$ is a dimensionless parameter and $U$ is an $\mathrm{SU}(2)$ matrix satisfying the boundary condition $\lim _{r \rightarrow \infty} U=I$ so that the pion field vanishes as $r$ goes to infinity. On the other hand, in the Skyrmion model, spin and isospin states can be treated by collective coordinates $a^{\mu}=\left(a^{0}, \vec{a}\right)(\mu=0,1,2,3)$ corresponding to the spin and isospin rotations

$$
A(t)=a^{0}+i \vec{a} \cdot \vec{\tau}
$$

With the hedgehog ansatz and the collective rotation $A(t) \in \mathrm{SU}(2)$, the Skyrmion Lagrangian can be written as

$$
L_{S M}=-E+2 \mathcal{I} \dot{a}^{\mu} \dot{a}^{\mu}
$$

where $E$ and $\mathcal{I}$ are the soliton energy and the moment of inertia, respectively $\mathbb{1}$, 5]. Introducing the canonical momenta $\pi^{\mu}=4 \mathcal{I} \dot{a}^{\mu}$ conjugate to the collective coordinates $a^{\mu}$ one can then obtain the canonical Hamiltonian

$$
H=E+\frac{1}{8 \mathcal{I}} \pi^{\mu} \pi^{\mu}
$$

Then, our system has the following second class constraints

$$
\begin{aligned}
& \Omega_{1}=a^{\mu} a^{\mu}-1 \approx 0 \\
& \Omega_{2}=a^{\mu} \pi^{\mu} \approx 0
\end{aligned}
$$

to yield the Poisson algebra with $\epsilon^{12}=-\epsilon^{21}=1$

$$
\Delta_{k k^{\prime}}=\left\{\Omega_{k}, \Omega_{k^{\prime}}\right\}=2 \epsilon^{k k^{\prime}} a^{\mu} a^{\mu} .
$$

Next, let us briefly recapitulate the construction of the first class SU(2) Hamiltonian. Following the abelian BFT formalism [4, 5] which systematically converts the second class constraints into first class ones, we introduce two auxiliary fields $\Phi^{i}$ corresponding to $\Omega_{i}$ with the Poisson brackets

$$
\left\{\Phi^{i}, \Phi^{j}\right\}=\epsilon^{i j} .
$$


One can then obtain the following first class constraints

$$
\begin{aligned}
& \tilde{\Omega}_{1}=\Omega_{1}+2 \Phi^{1} \\
& \tilde{\Omega}_{2}=\Omega_{2}-a^{\mu} a^{\mu} \Phi^{2}
\end{aligned}
$$

satisfying the first class constraint algebra $\left\{\tilde{\Omega}_{i}, \tilde{\Omega}_{j}\right\}=0$. Then, by demanding that they are strongly involutive in the extended phase space, i.e., $\left\{\tilde{\Omega}_{i}, \tilde{\mathcal{F}}\right\}=$ 0 , we construct the first class BFT physical fields $\tilde{\mathcal{F}}=\left(\tilde{a}^{\mu}, \tilde{\pi}^{\mu}\right)$ corresponding to the original fields $\mathcal{F}=\left(a^{\mu}, \pi^{\mu}\right)$, as a power series of the auxiliary fields $\Phi^{i}$, as follows

$$
\begin{aligned}
& \tilde{a}^{\mu}=a^{\mu}\left[1-\sum_{n=1}^{\infty} \frac{(-1)^{n}(2 n-3) ! !}{n !} \frac{\left(\Phi^{1}\right)^{n}}{\left(a^{\mu} a^{\mu}\right)^{n}}\right] \\
& \tilde{\pi}^{\mu}=\left(\pi^{\mu}-a^{\mu} \Phi^{2}\right)\left[1+\sum_{n=1}^{\infty} \frac{(-1)^{n}(2 n-1) ! !}{n !} \frac{\left(\Phi^{1}\right)^{n}}{\left(a^{\mu} a^{\mu}\right)^{n}}\right] .
\end{aligned}
$$

Now, exploiting the novel property 10, 11] that any functional $\mathcal{K}(\tilde{\mathcal{F}})$ of the first class fields $\tilde{\mathcal{F}}$ will also be first class, i.e.,

$$
\tilde{\mathcal{K}}(\mathcal{F} ; \Phi)=\mathcal{K}(\tilde{\mathcal{F}})
$$

one can directly construct the first class Hamiltonian in terms of the above BFT physical variables as follows

$$
\tilde{H}=E+\frac{1}{8 \mathcal{I}} \tilde{\pi}^{\mu} \tilde{\pi}^{\mu}
$$

omitting infinitely iterated standard procedure [5]. As a result, the corresponding first class Hamiltonian with the original fields and auxiliary fields is given by

$$
\tilde{H}=E+\frac{1}{8 \mathcal{I}}\left(\pi^{\mu}-a^{\mu} \Phi^{2}\right)\left(\pi^{\mu}-a^{\mu} \Phi^{2}\right) \frac{a^{\nu} a^{\nu}}{a^{\nu} a^{\nu}+2 \Phi^{1}}
$$

which is also strongly involutive with the first class constraints

$$
\left\{\tilde{\Omega}_{i}, \tilde{H}\right\}=0 .
$$

\footnotetext{
${ }^{1}$ Here one notes that the Poisson brackets of $\tilde{\mathcal{F}}$ 's have the same structure as that of the corresponding Dirac brackets 5 .
} 
However, with the first class Hamiltonian (2.11), one cannot naturally generate the first class Gauss' law constraint from the time evolution of the primary constraint $\tilde{\Omega}_{1}$. Now, by introducing an additional term proportional to the first class constraints $\tilde{\Omega}_{2}$ into $\tilde{H}$, we obtain an equivalent first class Hamiltonian

$$
\tilde{H}^{\prime}=\tilde{H}+\frac{1}{4 \mathcal{I}} \Phi^{2} \tilde{\Omega}_{2}
$$

which naturally generates the Gauss' law constraint

$$
\left\{\tilde{\Omega}_{1}, \tilde{H}^{\prime}\right\}=\frac{1}{2 \mathcal{I}} \tilde{\Omega}_{2}, \quad\left\{\tilde{\Omega}_{2}, \tilde{H}^{\prime}\right\}=0 .
$$

Here one notes that $\tilde{H}$ and $\tilde{H}^{\prime}$ act on physical states in the same way since such states are annihilated by the first class constraints.

It seems appropriate to comment on the phenomenolocal application of the above Hamiltonian $\tilde{H}^{\prime}$. Using the first class constraints in this Hamiltonian (2.13), one can finally obtain the Hamiltonian of the form

$$
\tilde{H}^{\prime}=M+\frac{1}{8 \mathcal{I}}\left(a^{\mu} a^{\mu} \pi^{\nu} \pi^{\nu}-a^{\mu} \pi^{\mu} a^{\nu} \pi^{\nu}\right)
$$

Following the symmetrization procedure, the first class Hamiltonian yields the slightly modified energy spectrum with the Weyl ordering correction [5]

$$
\left\langle\tilde{H}^{\prime}\right\rangle=E+\frac{1}{2 \mathcal{I}}\left[I(I+1)+\frac{1}{4}\right]
$$

where $I$ is the isospin quantum number of baryons.

\section{Symmetry Structure of First Class Hamil- tonian}

Now, since we have successfully converted the second class SU(2) Skyrmion into the corresponding first class one with the BFT scheme, we are ready to unravel gauge symmetries of the first class system following the purely Hamiltonian approach [6], which is very recently proposed. This total Hamiltonian approach $[$ is based on the requirement of the commutativity of a general

\footnotetext{
${ }^{2}$ For an extended Hamiltonian approach, see the work of [15]. This extended Hamiltonian approach with suitable gauge conditions is equivalent to the total Hamiltonian approach. See also Ref. [6].
} 
gauge variation with the time derivative operation which puts restrictions on gauge parameters and Lagrange multipliers.

Following Dirac's conjecture [3], let us first construct the generators of gauge transformation for the $\mathrm{SU}(2)$ Skyrmion model which has totally two constraints as

$$
G=\epsilon^{a} \tilde{\Omega}_{a}, \quad a=1,2 .
$$

Here, $\epsilon^{a}$ are in general functions of phase space variables and $\tilde{\Omega}_{a}$ are first class constraints in Eq.(2.8). Then, the infinitesimal gauge transformation is given by the relation of $\delta F(p, q)=\{F(p, q), G\}$ in which $F$ is a function of phase space variables. The total Hamiltonian is easily read as

$$
\tilde{H}_{\mathrm{T}}=\tilde{H}^{\prime}+\lambda \tilde{\Omega}_{1}
$$

where $\tilde{H}^{\prime}$ is the canonical Hamiltonian $\tilde{H}_{\mathrm{C}}$ and $\tilde{\Omega}_{1}$ the primary first class constraint. Comparing the general expression of gauge algebra

$$
\begin{aligned}
\left\{\tilde{\Omega}_{a}, \tilde{H}_{\mathrm{C}}\right\} & =V_{a}^{b} \tilde{\Omega}_{b}, \\
\left\{\tilde{\Omega}_{a}, \tilde{\Omega}_{b}\right\} & =C_{a b}^{c} \tilde{\Omega}_{c}
\end{aligned}
$$

with Eq. (2.14), we can determine the gauge functions of $V_{a}^{b}$ as well as $C_{a b}^{c}$. Note that the subscripts $a$ and $b$ count all the number of constraints, while denote the subscript of the primary constraints as $a_{1}$ and the secondary constraints as $a_{2}$.

Now the requirement of the commutativity of the general variation with the time derivative operator gives the relation [6] as follows

$$
\begin{aligned}
\delta v^{b_{1}} & =\frac{d \epsilon^{b_{1}}}{d t}-\epsilon^{a}\left(V_{a}^{b_{1}}+v^{a_{1}} C_{a_{1} a}^{b_{1}}\right), \\
0 & =\frac{d \epsilon^{b_{2}}}{d t}-\epsilon^{a}\left(V_{a}^{b_{2}}+v^{a_{1}} C_{a_{1} a}^{b_{2}}\right) .
\end{aligned}
$$

Here, $v^{b_{1}}$ are Lagrange multipliers associated with the primary first class constraints in the total Hamiltonian, and Eq.(3.5) gives the restriction on the gauge parameters.

Since there exist one primary and one secondary constraints for our SU(2) Skyrmion case, we easily see that the condition (3.5) imposed on the gauge parameters is simply rewritten as the relation of $\dot{\epsilon}^{2}=\frac{1}{2 \mathcal{I}} \epsilon^{1}$. Moreover, making 
use of this relation, we explicitly obtain the infinitesimal gauge transformation of the field variables as follows

$$
\delta a=\left\{a^{\mu}, G\right\}=a^{\mu} \epsilon, \quad \delta \Phi^{1}=\left\{\Phi^{1}, G\right\}=-a^{\mu} a^{\mu} \epsilon
$$

where we have rewritten the independent gauge paramether $\epsilon^{2}$ as $\epsilon$.

As a result of applying the approach on a purely Hamiltonian level to the $\mathrm{SU}(2)$ Skyrmion model, we have derived the rule of the full symmetry transformation.

\section{Symmetry Structure of Corresponding La- grangian}

Now let us consider the partition function of the model in order to present the Lagrangian corresponding to the first class Hamiltonian $\tilde{H}^{\prime}$ in Eq.(2.13). First of all we identify the auxiliary fields $\Phi^{i}$ with a canonical conjugate pair $\left(\theta, \pi_{\theta}\right)$, i.e.,

$$
\Phi^{i}=\left(\theta, \pi_{\theta}\right)
$$

which satisfy Eq. (2.7). Then, the starting partition function in the phase space is given by the Faddeev-Senjanovic formula [12] as follows

$$
\begin{aligned}
Z & =N \int \mathcal{D} a^{\mu} \mathcal{D} \pi^{\mu} \mathcal{D} \theta \mathcal{D} \pi_{\theta} \prod_{i, j=1}^{2} \delta\left(\tilde{\Omega}_{i}\right) \delta\left(\Gamma_{j}\right) \operatorname{det}\left|\left\{\tilde{\Omega}_{i}, \Gamma_{j}\right\}\right| e^{i \int \mathrm{d} t L} \\
L & =\dot{a}^{\mu} \pi^{\mu}+\dot{\theta} \pi_{\theta}-\tilde{H}^{\prime}
\end{aligned}
$$

where the gauge fixing conditions $\Gamma_{i}$ are chosen so that the determinant occurring in the functional measure is nonvanishing.

Now, exponentiating the delta function $\delta\left(\tilde{\Omega}_{2}\right)$ as $\delta\left(\tilde{\Omega}_{2}\right)=\int \mathcal{D} \xi e^{i \int \mathrm{d} t \xi \tilde{\Omega}_{2}}$ and performing the integration over $\pi_{\theta}, \pi^{\mu}$ and $\xi$, we obtain the the following partition function

$$
\begin{aligned}
Z & =N \int \mathcal{D} a^{\mu} \mathcal{D} \theta \delta\left(a^{\mu} a^{\mu}-1+2 \theta\right) \prod_{i=1}^{2} \delta\left(\Gamma_{i}\right) \operatorname{det}\left|\left\{\tilde{\Omega}_{i}, \Gamma_{j}\right\}\right| e^{i \int \mathrm{d} t L} \\
L & =-E+\frac{2 \mathcal{I}}{a^{\sigma} a^{\sigma}} \dot{a}^{\mu} \dot{a}^{\mu}-\frac{2 \mathcal{I}}{\left(a^{\sigma} a^{\sigma}\right)^{2}} \dot{\theta}^{2} .
\end{aligned}
$$


As a result, we have obtained the desired Lagrangian (4.4) corresponding to the first class Hamiltonian (2.13). Here one notes that the Lagrangian (4.4) can be reshuffled to yield the gauge invariant action of the form

$$
\begin{aligned}
S & =\int \mathrm{d} t\left(-E+2 \mathcal{I} \dot{a}^{\mu} \dot{a}^{\mu}\right)+S_{W Z} \\
S_{W Z} & =\int \mathrm{d} t\left[\frac{4 \mathcal{I}}{a^{\sigma} a^{\sigma}} \dot{a}^{\mu} \dot{a}^{\mu} \theta-\frac{2 \mathcal{I}}{\left(a^{\sigma} a^{\sigma}\right)^{2}} \dot{\theta}^{2}\right],
\end{aligned}
$$

where $S_{W Z}$ is the new type of the Wess-Zumino term restoring the gauge symmetry. Moreover the corresponding partition function (4.3) can be rewritten simply in terms of the first class physical fields (2.9)

$$
\begin{aligned}
\tilde{Z} & =N \int \mathcal{D} \tilde{a}^{\mu} \delta\left(\tilde{a}^{\mu} \tilde{a}^{\mu}-1\right) \prod_{i=1}^{2} \delta\left(\Gamma_{i}\right) \operatorname{det}\left|\left\{\tilde{\Omega}_{i}, \Gamma_{j}\right\}\right| \exp ^{i \int \mathrm{d} t \tilde{L}}, \\
\tilde{L} & =-E+2 \mathcal{I} \dot{\tilde{a}}^{\mu} \dot{\tilde{a}}^{\mu}
\end{aligned}
$$

where $\tilde{L}$ is form invariant Lagrangian of Eq. (2.3).

Next, in order to derive the exact form of transformation in which the Lagrangian (4.4) is invariant, we use the recently proposed method of Lagrangian approach [13, 14] which is based on a singular Hessian in the equations of motion. Starting with the Lagrangian (4.4) with the constraint $\tilde{\Omega}_{1}=a^{\mu} a^{\mu}-1+2 \theta=0$, we can obtain the equations of motion of the form

$$
L_{i}^{(0)}=W_{i j}^{(0)} \ddot{q}^{j}+\alpha_{i}^{(0)}=0
$$

where $W_{i j}^{(0)}=\frac{\partial^{2} \mathcal{L}}{\partial \dot{q}^{i} \dot{q}^{j}}$ is a Hessian, $\alpha_{i}^{(0)}=\frac{\partial^{2} \mathcal{L}}{\partial q^{j} \dot{q}^{i}} \dot{q}^{j}-\frac{\partial \mathcal{L}}{\partial q^{i}}$, and the superscript for later convenience means the zeroth iteration. If we denote $q^{i}=\left(a^{\mu}, \theta\right)$, we have

$$
\begin{aligned}
W_{i j}^{(0)} & =\frac{4 \mathcal{I}}{a^{\sigma} a^{\sigma}}\left(\begin{array}{cc}
\delta_{\mu \nu} & 0 \\
0 & -\frac{1}{a^{\sigma} a^{\sigma}}
\end{array}\right) \\
\alpha_{i}^{(0)} & =\frac{4 \mathcal{I}}{\left(a^{\sigma} a^{\sigma}\right)^{2}}\left(\begin{array}{c}
-2 \dot{a}_{\mu} a^{\rho} \dot{a}^{\rho}+a_{\mu} \dot{a}^{\rho} \dot{a}^{\rho}-\frac{2}{a^{\sigma} a^{\sigma}} a_{\mu} \dot{\theta}^{2} \\
\frac{4}{a^{\sigma} a^{\sigma}} a^{\rho} \dot{a}^{\rho} \dot{\theta}
\end{array}\right)
\end{aligned}
$$

Since the constraint $\Omega_{1}$ is an A-type defined by a function without velocities in configuration space, we require as a consistency condition the following identity

$$
L_{5} \equiv \frac{\mathrm{d}^{2}}{\mathrm{~d} t^{2}}\left(\frac{1}{2} \Omega_{1}\right)=a^{\mu} \ddot{a}^{\mu}+\ddot{\theta}+\dot{a}^{\mu} \dot{a}^{\mu}=0 .
$$


This requirement is similar to the time stability condition of constraints in the Hamiltonian formalism. Then, the resulting equation may be summarized in the form of the set of "first generation" equations

$$
L_{i_{1}}^{(1)} \equiv W_{i_{i} j}^{(1)} \ddot{q}^{j}+\alpha_{i_{1}}^{(1)}=\left\{\begin{array}{l}
L_{i}^{(0)}, i=a^{\mu}, \theta \\
\frac{\mathrm{d}^{2}}{\mathrm{~d} t^{2}}\left(\frac{1}{2} \Omega_{1}\right)
\end{array}\right.
$$

where

$$
W_{i_{1 j}}^{(1)}=\left(\frac{W_{i j}^{(0)}}{a^{\mu} 1}\right), \quad \alpha_{i_{1}}^{(1)}=\left(\begin{array}{c}
\alpha_{i}^{(0)} \\
\dot{a}^{\mu} \dot{a}^{\mu}
\end{array}\right) .
$$

Since the first iterated Hessian in Eq. (4.11) is of rank four, there exists a null eigenvector satisfying

$$
\lambda_{i_{1}}^{(1)} W_{i_{1} j}^{(1)}=0
$$

from which we can find the solution

$$
\lambda_{i_{1}}^{(1)}=\left(a^{\mu},-a^{\mu} a^{\mu},-\frac{4 \mathcal{I}}{a^{\mu} a^{\mu}}\right) .
$$

In general, the null eigenvectors are known to generate further Lagrange constraints which is of a function of the coordinates and velocities, but not of accelerations through $\lambda_{i_{k}}^{(k)} L_{i_{k}}^{(k)}=0$ at the $\mathrm{k}$-th generation of iteration. However, in our case we have

$$
\lambda_{i_{1}}^{(1)} L_{i_{1}}^{(1)}=-\frac{2 \mathcal{I}}{\left(a^{\sigma} a^{\sigma}\right)^{2}}\left(\frac{\mathrm{d}}{\mathrm{d} t} \Omega_{1}\right)^{2} \approx 0
$$

which means that no further constraints are generated. The algorithm is then ended up at this stage.

The symmetries of the Lagrangian (4.4) are encoded in the identity (4.14), which is a special case of a general theorem [13, 14 stating that the identity can always be written in the form of

$$
\Omega^{(l)}=\sum_{s=0}^{l}(-1)^{s} \frac{d^{s}}{d t^{s}} \phi_{k}^{i(s)} L_{i}^{(0)} \approx 0
$$

where the superscript $l$ denotes the last stage of iteration giving the identity. The corresponding Lagrangian is then invariant under the transformation

$$
\delta \varphi^{i}=\sum_{k}\left(\epsilon_{k} \phi_{k}^{i(0)}+\dot{\epsilon}_{k} \phi_{k}^{i(1)}\right) .
$$


In the first class $\mathrm{SU}(2)$ Skyrmion, the coefficients $\phi^{i(s)}$ in Eq. (4.15) are given by $\phi^{a^{\mu}(0)}=a^{\mu}, \phi^{\theta(0)}=-a^{\mu} a^{\mu}$. As results, by using Eq. (4.16), the desired form of symmetry transformation can be read as

$$
\delta a^{\mu}=a^{\mu} \epsilon, \quad \delta \theta=-a^{\mu} a^{\mu} \epsilon .
$$

It can be easily checked that the Lagrangian (4.4) is invariant under the transformation (4.17).

Therefore we have shown that both the Hamiltonian and Lagrangian approaches have the same symmetry structure because the symmetry transformation rule (4.17) obtained in the Lagrangian approach is exactly the same as that in Eq. 3.6) obtained when we consider the effective first class constraints (2.8) as the symmetry generators in the pure Hamiltonian formalism.

\section{BRST-BFV analysis for consistent gauge fixing}

Now, in order to obtain the BRST invariant Lagrangian in the framework of the BFV formalism [7, 8, 9] which is applicable to theories with the first class constraints, we introduce two canonical sets of ghosts and anti-ghosts together with auxiliary fields $\left(\mathcal{C}^{i}, \overline{\mathcal{P}}_{i}\right),\left(\mathcal{P}^{i}, \overline{\mathcal{C}}_{i}\right),\left(N^{i}, B_{i}\right),(i=1,2)$ which satisfy the super-Poisson algebra ${ }^{\beta}$

$$
\left\{\mathcal{C}^{i}, \overline{\mathcal{P}}_{j}\right\}=\left\{\mathcal{P}^{i}, \overline{\mathcal{C}}_{j}\right\}=\left\{N^{i}, B_{j}\right\}=\delta_{j}^{i}
$$

In the $\mathrm{SU}(2)$ Skyrmion model, the nilpotent BRST charge $Q$, the fermionic gauge fixing function $\Psi$ and the BRST invariant minimal Hamiltonian $H_{m}$ are given by

$$
\begin{aligned}
Q & =\mathcal{C}^{i} \tilde{\Omega}_{i}+\mathcal{P}^{i} B_{i} \\
\Psi & =\overline{\mathcal{C}}_{i} \chi^{i}+\overline{\mathcal{P}}_{i} N^{i} \\
H_{m} & =\tilde{H}^{\prime}-\frac{1}{2 \mathcal{I}} \mathcal{C}^{1} \overline{\mathcal{P}}_{2}
\end{aligned}
$$

${ }^{3}$ Here the super-Poisson bracket is defined as

$$
\{A, B\}=\left.\left.\frac{\delta A}{\delta q}\right|_{r} \frac{\delta B}{\delta p}\right|_{l}-\left.\left.(-1)^{\eta_{A} \eta_{B}} \frac{\delta B}{\delta q}\right|_{r} \frac{\delta A}{\delta p}\right|_{l}
$$

where $\eta_{A}$ denotes the number of fermions called ghost number in $A$ and the subscript $r$ and $l$ right and left derivatives. 
which satisfy the relations $\left\{Q, H_{m}\right\}=0, Q^{2}=\{Q, Q\}=0,\{\{\Psi, Q\}, Q\}=0$. The effective quantum Lagrangian is then described as

$$
L_{\text {eff }}=\pi^{\mu} \dot{a}^{\mu}+\pi_{\theta} \dot{\theta}+B_{2} \dot{N}^{2}+\overline{\mathcal{P}}_{i} \dot{\mathcal{C}}^{i}+\overline{\mathcal{C}}_{2} \dot{\mathcal{P}}^{2}-H_{t o t}
$$

with $H_{\text {tot }}=H_{m}-\{Q, \Psi\}$. Here $B_{1} \dot{N}^{1}+\overline{\mathcal{C}}_{1} \dot{\mathcal{P}}^{1}=\left\{Q, \overline{\mathcal{C}}_{1} \dot{N}^{1}\right\}$ terms are suppressed by replacing $\chi^{1}$ with $\chi^{1}+\dot{N}^{1}$.

Now we choose the unitary gauge

$$
\chi^{1}=\Omega_{1}, \quad \chi^{2}=\Omega_{2}
$$

and perform the path integration over the fields $B_{1}, N^{1}, \overline{\mathcal{C}}_{1}, \mathcal{P}^{1}, \overline{\mathcal{P}}_{1}$ and $\mathcal{C}^{1}$, by using the equations of motion, to yield the effective Lagrangian of the form

$$
\begin{aligned}
L_{e f f}= & \pi^{\mu} \dot{a}^{\mu}+\pi_{\theta} \dot{\theta}+B \dot{N}+\overline{\mathcal{P}} \dot{\mathcal{C}}+\overline{\mathcal{C}} \dot{\mathcal{P}} \\
& -E-\frac{1}{8 \mathcal{I}}\left(\pi^{\mu}-a^{\mu} \pi_{\theta}\right)\left(\pi^{\mu}-a^{\mu} \pi_{\theta}\right) \frac{a^{\sigma} a^{\sigma}}{a^{\sigma} a^{\sigma}+2 \theta}-\frac{1}{4 \mathcal{I}} \pi_{\theta} \tilde{\Omega}_{2} \\
& +2 a^{\mu} a^{\mu} \pi_{\theta} \overline{\mathcal{C}} \mathcal{C}+\tilde{\Omega}_{2} N+B \Omega_{2}+\overline{\mathcal{P}} \mathcal{P}
\end{aligned}
$$

with redefinitions: $N \equiv N^{2}, B \equiv B_{2}, \overline{\mathcal{C}} \equiv \overline{\mathcal{C}}_{2}, \mathcal{C} \equiv \mathcal{C}^{2}, \overline{\mathcal{P}} \equiv \overline{\mathcal{P}}_{2}, \mathcal{P} \equiv \mathcal{P}_{2}$.

Next, using the variations with respect to $\pi^{\mu}, \pi_{\theta}, \mathcal{P}$ and $\overline{\mathcal{P}}$, one obtain the relations

$$
\begin{aligned}
\dot{a}^{\mu} & =\frac{1}{4 \mathcal{I}}\left(\pi^{\mu}-a^{\mu} \pi_{\theta}\right) a^{\sigma} a^{\sigma}+a^{\mu}\left(\frac{1}{4 \mathcal{I}} \pi_{\theta}-N-B\right) \\
\dot{\theta} & =-\frac{1}{4 \mathcal{I}} a^{\mu}\left(\pi^{\mu}-a^{\mu} \pi_{\theta}\right) a^{\sigma} a^{\sigma}+a^{\mu} a^{\mu}\left(-\frac{1}{2 \mathcal{I}} \pi_{\theta}-2 \overline{\mathcal{C}} \mathcal{C}+N\right)+\frac{1}{4 \mathcal{I}} a^{\mu} \pi^{\mu} \\
\mathcal{P} & =-\dot{\mathcal{C}}, \quad \overline{\mathcal{P}}=\dot{\overline{\mathcal{C}}}
\end{aligned}
$$

to yield the effective Lagrangian

$$
\begin{aligned}
L_{e f f}= & -E+\frac{2 \mathcal{I}}{a^{\sigma} a^{\sigma}} \dot{a}^{\mu} \dot{a}^{\mu}-2 \mathcal{I}\left[\frac{\dot{\theta}}{a^{\sigma} a^{\sigma}}+(B+2 \overline{\mathcal{C}} \mathcal{C}) a^{\sigma} a^{\sigma}\right]^{2} \\
& +\frac{4 \mathcal{I}}{a^{\sigma} a^{\sigma}} a^{\mu}\left[\dot{a}^{\mu}+a^{\mu}\left(\frac{\dot{\theta}}{a^{\sigma} a^{\sigma}}+(B+2 \overline{\mathcal{C}} \mathcal{C}) a^{\sigma} a^{\sigma}\right)\right](B+N) \\
& +B \dot{N}+\dot{\overline{\mathcal{C}}} \dot{\mathcal{C}} .
\end{aligned}
$$


Finally, with the identification

$$
N=-B+\frac{\dot{\theta}}{a^{\sigma} a^{\sigma}}
$$

we obtained the desired BRST invariant Lagrangian of the form

$$
\begin{aligned}
L_{e f f}= & -E+\frac{2 \mathcal{I}}{a^{\sigma} a^{\sigma}} \dot{a}^{\mu} \dot{a}^{\mu}-\frac{2 \mathcal{I}}{\left(a^{\sigma} a^{\sigma}\right)^{2}} \dot{\theta}^{2}-2 \mathcal{I}\left(a^{\mu} a^{\mu}\right)^{2}(B+2 \overline{\mathcal{C}} \mathcal{C})^{2} \\
& -\frac{\dot{\theta} \dot{B}}{a^{\sigma} a^{\sigma}}+\dot{\overline{\mathcal{C}}} \dot{\mathcal{C}},
\end{aligned}
$$

which is invariant under the BRST transformation

$$
\begin{aligned}
\delta_{B} a^{\mu} & =\lambda a^{\mu} \mathcal{C}, \quad \delta_{B} \theta=-\lambda a^{\mu} a^{\mu} \mathcal{C}, \\
\delta_{B} \overline{\mathcal{C}} & =-\lambda B, \quad \delta_{B} \mathcal{C}=\delta_{B} B=0 .
\end{aligned}
$$

Here one notes that the above BRST transformation including the rules for the (anti)ghost fields is just the generalization of the previous one (4.17).

This completes the standard procedure of BRST invariant gauge fixing in the BFV formalism.

\section{Conclusions}

In summary, we have constructed the first class BFT physical fields, in terms of which the first class Hamiltonian is formulated to be consistent with the Hamiltonian with the original fields and auxiliary fields. After converting the second class SU(2) Skyrmion into the effectively first class one, we have analyzed the full symmetry structure of the model purely based on the Hamiltonian approach. On the other hand, we have also constructed the effective Lagrangian corresponding to the first class Hamiltonian in the path integral approach to the partition function. This Lagrangian includes the new type of the WZ term restoring the gauge symmetry. Then, we have explicitly derived the symmetry structure of this effective Lagrangian through the Lagrangian approach, showing that the two approaches are equivalent to give the same symmetry structure of the SU(2) Skyrmion. Furthermore, in the BFV scheme, we have obtained the BRST invariant gauge fixed Lagrangian including the (anti)ghost fields, and its BRST transformation rules. Finally, 
through further investigation, the $\mathrm{SU}(3)$ extension [16], which is a real phenomenolical model, of this analysis will be worth to being studied.

One of us (S.T.H.) would like to thank Professor G.E. Brown at Stony Brook for constant concerns and encouragement. The present work was partly supported by the BK21 Project No. D-0055, Ministry of Education, 1999 and the Sogang University Research Grants in 1999.

\section{References}

[1] G.S. Adkins, C.R. Nappi, E. Witten, Nucl. Phys. B228, 552 (1983).

[2] I. Zahed, G.E. Brown, Phys. Rep. 142, 1 (1986); S.-T. Hong, Phys. Lett. B417, 211 (1998).

[3] P.A.M. Dirac, Lectures in Quantum Mechanics (Yeshiva University, New York, 1964).

[4] I.A. Batalin, E.S. Fradkin, Phys. Lett. B180, 157 (1986); Nucl. Phys. B279, 514 (1987); I.A. Batalin, I.V. Tyutin, Int. J. Mod. Phys. A6, 3255 (1991).

[5] S.-T. Hong, Y.-W. Kim, Y.-J. Park, Phys. Rev. D59, 114026 (1999).

[6] R. Banerjee, H. J. Rothe and K. D. Rothe, Phys. Lett. B463, 248 (1999); hep-th/9907217; hep-th/9909039.

[7] E.S. Fradkin, G.A. Vilkovisky, Phys. Lett. B55, 224 (1975); M. Henneaux, Phys. Rep. C126, 1 (1985).

[8] T. Fujiwara, Y. Igarashi, J. Kubo, Nucl. Phys. B341, 695 (1990); Y.-W. Kim, S.-K. Kim, W. T. Kim, Y.-J. Park, K. Y. Kim, Y. Kim, Phys. Rev. D46, 4574 (1992).

[9] C. Bizdadea, S.O. Saliu, Nucl. Phys. B456, 473 (1995); S. Hamamoto, Prog. Theor. Phys. 95, 441 (1996). 
[10] Y.-W. Kim, M.-I. Park, Y.-J. Park, S.J. Yoon, Int. J. Mod. Phys. A12, 4217 (1997).

[11] Y.-W. Kim, Y.-J. Park, K.D. Rothe, J. Phys. G24, 953 (1998); Y.-W. Kim, K. D. Rothe, Nucl. Phys. B510, 511 (1998); Y.-W. Kim, Y.-J. Park, Mod. Phys. Lett. A13, 1201 (1998).

[12] L.D. Faddeev, Theor. Math. Phys. 1, 1 (1970); P. Senjanovich, Ann. Phys. (N.Y.) 100, 277 (1976).

[13] A. Shirzad, J. Phys. A31, 2747 (1998); Y.-W. Kim, K. D. Rothe, Int. J. Mod. Phys. A13, 4183 (1998).

[14] S.-K. Kim, Y.-W. Kim, Y.-J. Park, Mod. Phys. Lett. A13, 2653 (1998); J. Phys. A31, 8677 (1998).

[15] M. Henneaux, C. Teitelboin and J. Zanelli, Nucl. Phys. B332, 169 (1990).

[16] E. Guadagnini, Nucl. Phys. B236, 35 (1984); P. Mazur, M. Nowak, and M. Praszalowicz, Phys. Lett. B147, 137 (1984); A. Manohar, Nucl. Phys. B248, 19 (1984); M. Chemtob, Nucl. Phys. B256, 600 (1985); K.M. Westerberg, and I.R. Klebanov, Phys. Rev. D50, 5834 (1994). 